\title{
Hacia una historia de la lucha antituberculosa en Colombia
}

\section{Jorge Márquez* Oscar Gallo**}

\section{Resumen}

El problema de la historia social y cultural de la lucha antituberculosa en Colombia apenas se ha planteado por los investigadores. Una lectura cruzada de cifras y discursos sobre la tuberculosis de finales del siglo XIX y comienzos del siglo XX ayudaría a responder la pregunta: ¿se trató de un problema sanitario colectivo, en términos biomédicos y epidemiológicos, o conformó solo un conjunto de representaciones sociales y culturales basadas en los estigmas atribuidos a esta enfermedad? En cuanto a la historiografía, este artículo evalúa lo que se ha hecho en este campo en Colombia para proponer nuevas estrategias de investigación que inscriban la lucha antituberculosa como problema sociocultural en la historia de las políticas de salud y de las reformas sanitarias en Colombia entre 1913 y 1950. En cuanto al archivo, se hace énfasis en la documentación publicada y de archivos sobre campañas, prevención, terapéutica, higiene, clínica y debate eugenista.

Palabras-clave: historia de la tuberculosis-Colombia, procesos de medicalización, representación social de la enfermedad, historia de la medicina, historia de la medicina-Colombia.

\footnotetext{
Profesor asociado del Departamento de Estudios Filosóficos de la Facultad de Ciencias Humanas y Económicas de la Universidad Nacional de Colombia, sede Medellín. Director del Grupo de Investigación Producción, Circulación y Apropiación de Saberes - PROCIRCAS. Correo electrónico: jmarquez@unal.edu.co

**: Estudiante del doctorado en Historia de la Universidad Federal de Santa Catarina (Florianópolis, Brasil). Becario de la Coordenação de Aperfeiçoamento de Pessoal de Nível Superior (CAPES). Integrante del Grupo de Investigación Producción, Circulación y Apropiación de Saberes, PROCIRCAS (Colombia) y del Laboratório de História, Saúde e Sociedade - LABHISS (Brasil). Correo electrónico: oscargallovelez@gmail.com.
} 


\section{Discusión historiográfica}

a historiografía de la tuberculosis en Colombia es escasa. ${ }^{1}$ HeLmos recogido lo que se ha hecho hasta ahora en historia de la tuberculosis en Colombia mediante dos métodos: Primero, por la consulta exhaustiva de los catálogos de bibliotecas y las bases de datos disponibles. Segundo, formulando la pregunta sobre la existencia de trabajos publicados a la mayoría de los investigadores que actualmente se interesan en este problema. De todas maneras, es posible que el inventario así obtenido no sea un resultado exhaustivo, pero es muy representativo, pues contiene los trabajos de mayor visibilidad así como las tesis y monografías universitarias.

Por otra parte, no todos los trabajos citados tratan directamente el problema de la historia de la tuberculosis en Colombia. Por ejemplo, el primer estudio que pone en relación condiciones de vida, problemas sociales y presencia de la tuberculosis es el del médico salubrista y doctor en historia, Álvaro Cardona Saldarriaga (1984), aunque la tuberculosis es solo uno entre los diversos problemas de salud de la Colombia de la primera mitad del siglo XX que estudia.

El médico y especialista en infectología, Sigifredo Ospina, publicó en 2001 un artículo relacionado con la historia general de la tuberculosis en donde enumera los hitos de la historia natural de la enfermedad, los de la historia del conocimiento médico de ella y algunos acontecimientos legislativos de la lucha antituberculosa en Colombia (OSPINA, 2001). Lo importante de este artículo es el balance crítico que hace del panorama epidemiológico de la tuberculosis en Colombia a finales del siglo XX.

En un artículo de 2001, del médico y epidemiólogo Álvaro Javier Idrovo, hay preguntas históricas sobre la presencia de la tuberculosis en Bogotá a finales del siglo XIX y comienzos del siglo $\mathrm{XX}$. Es importante este aporte porque, basado en fuentes histó-

1 Lo poco que se ha hecho en historia, hasta donde sabemos, sea que trate directa o indirectamente el problema de la historia de la tuberculosis en Colombia, lo enumeramos en un anexo de bibliografía crítica al final de este artículo. 
ricas, el autor interroga la existencia de una epidemia de tuberculosis en la ciudad capital durante la transición del siglo XIX al siglo XX (IDROVO, 2001). En otro artículo de 2004, este mismo investigador indaga sobre el comportamiento epidemiológico de la tuberculosis desde tiempos prehispánicos hasta el siglo XX en territorio colombiano. En el artículo hay afirmaciones contundentes sobre la existencia de una epidemia de tuberculosis en Bogotá a comienzos del siglo XX, así como sobre la de una endemia nacional en el mismo periodo, pero el autor no aportó el material probatorio para sustentarlas. Él mismo afirma que: "Conocer con exactitud cuál fue la ocurrencia de TBC en Bogotá entre 1870 y 1920 no es una terea fácil, debido a la ausencia de unas adecuadas estadísticas" (IDROVO, 2004, p. 217). Y confiesa que él mismo conoce una única fuente, los archivos del Hospital san Juan de Dios (IDROVO, 2004, p. 217). Los aportes de estos dos artículos de Idrovo tienen que ver más con la epidemiología que con la historia de la enfermedad en Colombia.

Un artículo de Magnolia Arango Loboguerrero de 2003 señala algunos hitos mundiales de la historia de la tuberculosis infantil. En este trabajo, como en los ya citados de Idrovo y de Ospina, los datos históricos son instrumentalizados para argumentar a favor de la investigación epidemiológica de la tuberculosis. De los artículos hasta aquí evocados hay que destacar que enriquecen una cultura general sobre la tuberculosis, pero casi no aportan nuevos conocimientos a la historia social y cultural de la tuberculosis en Colombia (LOBOGUERRERO, 2003).

Las pocas investigaciones hechas desde el campo de la historia se circunscriben a ciertas ciudades como Bogotá y Medellín o a determinadas instituciones durante periodos precisos. Pero es en ellas donde ha sido planteada la historia de la tuberculosis en Colombia en su complejidad, es decir como un problema cuyo aspecto biomédico es solo una de las facetas. En la historiografía especializada, estos trabajos universitarios que podemos llamar propiamente históricos se distinguen porque responden a pregun- 
tas planteadas por la historia como campo de investigación²: ¿Cuál ha sido la respuesta oficial e institucional frente a la tuberculosis como problema social y enfermedad colectiva? ¿Qué relaciones han podido existir entre flujos migratorios, industrialización, urbanización y tuberculosis? ¿Cómo se ha percibido y cómo se ha representado la tuberculosis en la sociedad colombiana en cada periodo de su historia?

Entre los trabajos propiamente históricos se destaca la tesis de maestría en historia de la médica neumóloga Magnolia Arango (2007), porque estudia en detalle las estrategias de control y las representaciones que la tuberculosis suscitó en la sociedad bogotana del cambio del siglo XIX al XX.

Mariela Vélez Alzate (2001) y María Bernarda Ocampo (2005) hicieron sendas monografías para obtener el título de historiadoras y ambas trabajaron sobre el primer sanatorio de tuberculosis creado en Colombia, el Hospital La María, proyectado desde 1920 por el Concejo municipal de Medellín ${ }^{3}$ e inaugurado en 1923. Mientras que Vélez se limita a presentar una relación descriptiva de algunos aspectos institucionales, Ocampo inscribe la creación del nuevo Hospital en los proyectos higienistas y de medicalización urbana del Medellín de comienzos del siglo XX. En otra monografía de historia, sobre "las enfermedades sociales en los obreros de Medellín, 1900-1930" (2007), Jana Catalina Congote dedica un capítulo a la tuberculosis, pero se limita a una lectura descriptiva de la tesis de medicina de Jesús María Duque (1889).

Andrés Renet Arango, en su monografía de historia (2010), ha presentado resultados de investigación sobre la historia de la lucha antituberculosa en Medellín a comienzos del siglo XX. La

2 Independientemente de si los autores son o no historiadores profesionales, lo que interesa aquí es precisar si las preguntas se inscriben en las tendencias historiográficas de los siglos XX y XXI, es decir de la disciplina histórica como campo de investigaciones.

3 La Junta Municipal Organizadora de la Lucha Antituberculosa fue creada por el Acuerdo ${ }^{\circ}$ 4 de enero de 1920 (CRÓNICA MUNICIPAL, 1920, p. 21). Entre sus tareas estratégicas, la principal fue la de coordinar y propiciar la construcción de un sanatorio de montaña para Medellín. Este fue inaugurado el 12 de Octubre de 1923 (CRÓNICA MUNICIPAL, 1923, p. 3023). 
originalidad de este trabajo radica en su punto de partida constituido por preguntas nunca antes formuladas por la historiografía. Se interroga por las relaciones entre las estrategias sanitarias, la estadística de la enfermedad y el funcionamiento del primer sanatorio creado en el país. Junto con la tesis de maestría en historia de Magnolia Arango constituyen los dos únicos trabajos que asumen el problema de la historia de la tuberculosis en Colombia en su complejidad, es decir como problema a la vez social, institucional, político, biomédico, económico y cultural.

Una característica común a la tesis de maestría y a las cuatro monografías de historia citadas -lo que constituye su novedad- es la interrogación, desde varios puntos de vista, sobre la estrategia sanitaria englobada en la historia de Colombia bajo el nombre de "lucha antituberculosa".

También desde el punto de vista de la historia de las instituciones, hay que señalar el artículo de Mario Hernández y Héctor Maldonado (2004) que describe la creación del pabellón para tuberculosos en el Hospital San Carlos de Bogotá. Resume la existencia de ese hospital como sanatorio antituberculoso: orígenes, aspectos arquitectónicos, conceptos científico-médicos de un sanatorio en la "era preantibiótica", cambios debidos a la introducción de la quimioterapia antibiótica y a sus efectos en la desaparición de la cura sanatorial en Bogotá. Lo más valioso de este artículo es su descripción de la evolución de las terapéuticas antituberculosas en el caso de una región colombiana.

Finamente, en cuanto a la historia natural de la enfermedad ha habido valiosos aportes de investigadores colombianos: Álvaro Javier Idrovo (1997); Hugo Sotomayor, Javier Burgos y Magnolia Arango (2004); Abel Martínez Martín, Bernardo Meléndez Álvarez y Leidy Gamboa Gamboa (2006); José Rodríguez Cuenca (2006). En estos trabajos de paleopatología se presentan evidencias sobre la presencia de la tuberculosis en el mundo precolombino, en diversas localidades geográficas del actual territorio colombiano.

De este balance historiográfico se desprenden muchas más preguntas que resultados de investigación. Las características de 
esta historiografía son el escaso número de trabajos, la dispersión, el carácter fragmentario, monográfico, descriptivo y poco analítico. A partir de este recorrido es evidente que ha habido desatención por parte de los historiadores frente a la historia de la tuberculosis en Colombia. Esta situación plantea varios interrogantes: ¿será que se ha considerado la tuberculosis como un problema histórico menor? ¿En algo habrá incidido el obstáculo de la gran cantidad y dispersión de fuentes impresas y de archivo? ¿O quizás la prevalencia de esta enfermedad en Colombia, durante los siglos XIX y XX, ha sido difícil de medir, y las mediciones que hay la muestran como un mal menor, si se lo compara con el paludismo, las enfermedades gastrointestinales y el hambre? ¿Se puede ligar esta ausencia a una situación general de precariedad en la historia de la enfermedad y la salud en Colombia?

Es difícil responder inmediatamente estas preguntas, pero se puede avanzar por ahora que quizás en este olvido también influya que algunos historiadores se detengan ante el prejuicio según el cual los problemas de la historia de las enfermedades deben ser estudiados por médicos, y que por lo tanto habría que seguir esperando algún pronunciamiento por parte de ellos.

Los estudios realizados para Europa (GRMEK, 1999), Estados Unidos (TELLER, 1988), Francia (GUILLAUME, 1986; 1988), Inglaterra (BRYDER, 1988), España (MOLERO MESA, 1987; RODRÍGUEZ OCAÑA, 1992), Argentina (CARBONETTI, 1988; ARMUS, 2007) y Brasil (NASCIMENTO, 2008; PORTO, 2008; SILVEIRO; NASCIMENTO, 2004) - por mencionar sólo algunos de los más agudos-, muestran que la tuberculosis, como hecho social y cultural, es decir como enfermedad dominante de cierto periodo de la historia de la humanidad, es resultado de una confluencia de acontecimientos de muy diversa índole que acaecieron entre finales del siglo XVIII y la Segunda Guerra mundial. Ese periodo coincide con inmensos avances en el campo del saber médico en general y en el del conocimiento de la tuberculosis en particular: la perfecta objetivación anatomo-clínica lograda desde finales del siglo XVIII por autores como Desault, Dupré de Lisle o Rozière; la "auscultación media- 
ta” de Laennec (1819) y su unificación de las múltiples manifestaciones de la enfermedad; la inoculación infecciosa en animales, lograda por J. A. Villemin, en 1865; el aislamiento del bacilo por Robert Koch, en 1882; las posibilidades diagnósticas de comienzos del siglo XX, tales como la aplicación de los rayos X descubiertos por Karl Rötngen en 1895 y la cuti-reacción descubierta por Von Pirquet en 1907, y el descubrimiento de los antibióticos (1940) y la aplicación de la estreptomicina desde $1944 .{ }^{4}$

Por otro lado, los estudios citados dejan claro que se trata de un verdadero "mal del siglo", no en el sentido literario, sino en el sentido de la ubicación patocenótica ${ }^{5}$ de la tuberculosis como endemia ligada a cambios drásticos en las condiciones de vida de las poblaciones debidos a su vez a procesos acelerados de urbanización e industrialización.

La calidad y la profundidad de los estudios hechos para otros casos ponen aun más en evidencia la ausencia de investigaciones sobre el caso de Colombia, donde el problema no se ha estudiado todavía para el ámbito nacional. Según los pocos estudios parciales existentes y según las fuentes de archivo, es evidente que una estrategia oficial denominada "lucha antituberculosa" (1916-1935) y

4 En 1944 se produjo un acontecimiento que constituye lo que se ha llamado la mayor revolución terapéutica de la historia. En New-Jersey, Selman A. Waksman y sus alumnos pusieron en evidencia la acción antibiótica de amplio espectro de la estreptomicina, molécula extraída del hongo Streptomices griseus. Este descubrimiento, fundado en el antagonismo microbiano entre bacilo tuberculoso y Streptomices griseus, hizo merecedor a Waksman del premio Nobel en 1952. Centre Médical de Bligny. Bligny et son histoire: du sanatorium au centre médical d'aujourd'hui. Document numérique du Centre Médical de Bligny (2008).

5 En cuanto a los conceptos de "patocenosis"y de "enfermedad dominante", los historiadores Mirko D. Grmek y Jean-Charles Sournia afirman: "Las enfermedades que golpean a una población determinada durante un periodo histórico dado constituyen un conjunto llamado patocenosis. Se trata de un conjunto estructurado en el cual la repartición de las frecuencias de diversas enfermedades presenta ciertas características matemáticas. De esta manera, en toda patocenosis histórica existe un pequeño número de enfermedades muy frecuentes, de morbilidad extremadamente elevada, y existe también un gran número de enfermedades relativamente raras. Consideramos como dominantes las enfermedades a la vez muy frecuentes y que comportan consecuencias graves para la situación demográfica y la calidad de vida de una población general o de una subpoblación determinada (GRMEK; SOURNIA, 1999, p. 271-272; trad. libre del autor). 
otra denominada "campaña nacional contra la tuberculosis" (19351959) tuvieron cierto despliegue y alguna incidencia en el conocimiento de la endemia en el país, y jugaron quizás algún papel en su retroceso. Sin embargo, la situación historiográfica colombiana es tan precaria en este campo que es necesario comenzar por preguntar si la tuberculosis ha existido realmente como endemia y como epidemia en Colombia. Para esbozar una respuesta a esta pregunta habría que comenzar por un examen crítico de las huellas que esta enfermedad ha dejado en nuestra memoria colectiva y en nuestros archivos.

Un primer examen nos muestra evidencias de que, históricamente, la tuberculosis ha sido realmente un problema sanitario para la sociedad colombiana. En los discursos médicos del siglo XIX y de la primera mitad del siglo XX esta enfermedad aparece casi siempre como un problema muy acucioso y amenazante para la sociedad, al mismo tiempo que largamente descuidado por parte de las autoridades políticas.

Es innegable que este problema histórico hay que estudiarlo en todas sus dimensiones: simbólica, social, política y biomédica. Varios historiadores (QUEVEDO, 1993; OBREGÓN, 2002) han mostrado que, en historia de las enfermedades, al enfocarse sólo en la dimensión biomédica, el investigador se acerca a uno solo de los aspectos del problema, aunque no al menos importante. Inversamente, al olvidar los aspectos cambiantes de la dimensión biomédica, el historiador pierde la oportunidad de estudiar los grandes problemas de la historia de las enfermedades, los que afectan el curso de las civilizaciones (GRMEK, 1969). Entre esos problemas están los que han influido particularmente en el devenir de nuestra relación con la tuberculosis. Un ejemplo notable es el de la prolongada impotencia terapéutica contra ella: casi ochenta años (entre 1865 y 1945) de lucha denodada contra la enfermedad (estrategias higienistas, sanatoriales y quirúrgicas) y casi nada en recursos terapéuticos eficaces. 


\section{La tuberculosis como fenómeno social y cultural}

Un primer obstáculo que se enfrenta al intentar hacer historia de la tuberculosis con herramientas actuales se halla en la concepción relativista extrema de algunos científicos sociales, que pretenden enfrentar el objeto enfermedad no como una realidad resultado de relaciones bio-ecológicas e históricas (por ende, bio-socio-culturales), sino sobre todo como una representación social y cultural.

A partir de la luminosa idea del filósofo de las ciencias Ludwik Fleck (1986; 2010), de estudiar las enfermedades no solamente como realidades biológicas sino también como realidades sociales y culturales, ciertos investigadores se han deslizado hacia una forma ideológica de concebir la enfermedad, según la cual se cree que su dimensión simbólica es la realidad que merece ser estudiada por las ciencias sociales. Nunca fue tan ingenuo Ludwik Fleck como para creer que nuestras relaciones con la sífilis o con el tifus ${ }^{6}$ fueran solamente biológicas o solamente "construcciones" culturales. La confusión proviene, en parte, de la creencia de algunos historiadores en que la sociología de las ciencias puede desplazar, aplazar o relegar, en historia, toda pregunta epistemológica, o de que para todo cambio epistemológico tiene que haber forzosamente una explicación sociológica o, al menos, psicológica. Como si toda explicación de un acontecimiento, una corriente de pensamiento, o una transformación en los conceptos debiera tener fundamentos en el devenir social, cultural y personal de los individuos o de las colectividades implicadas. Es evidente que pueden existir relaciones entre la configuración social, cultural y espacial de un grupo humano, las enfermedades que lo aquejan y sus formas de concebir la enfermedad, en un momento determinado de su historia, pero esas relaciones no necesariamente tienen que ser de causa a efecto.

6 Sífilis y tifus: dos enfermedades cuyas objetivaciones científicas Fleck aprovechó lúcidamente en su teoría de la dimensión social de la actividad científica (FLECK, 1986) y (FLECK, 1988). 
La actitud psicologizante y sociologizante hace evocar inevitablemente un ejemplo clásico - aunque un poco olvidado - de abuso de relativismo en la aplicación de la sociología a la historia. En 1998, como una continuidad de su defensa militante del relativismo epistemológico, el sociólogo Bruno Latour (1998) publicó un artículo en la página de debate y opinión que él dirigía en una importante revista de divulgación de las ciencias. En él se refiere al descubrimiento, en 1976, por parte de arqueólogos forenses, que estudiaban mediante autopsia la momia del faraón Ramsés II (siglo XIII a.C.), de la presencia de huellas del bacilo de Koch y de lesiones de tuberculosis. Latour preguntaba: ¿cómo es posible que muriera Ramsés II de una bacteria descubierta por Robert Koch en 1882 ? Y señaló el descubrimiento como un verdadero anacronismo. El sociólogo confundió realidad biológica y realidad cultural. Aunque aceptó que el sentido común puede permitirnos pensar que Robert Koch descubrió una bacteria que ha convivido con la humanidad quizás durante miles de años, rechazó de manera contradictoria que la paleopatología pudiera enunciar esa causa de enfermedad y quizás de muerte en una persona momificada hace más de veinte siglos. En su artículo no ofrece argumento alguno para sostener su posición, no aporta ningún punto de vista claro y corre el riesgo de incitar a despreciar por "anacrónicas" las preguntas paleopatológicas que, sin embargo, no son para nada ociosas. Ellas son del tipo: ¿Existía tuberculosis en la antiguiedad? ¿Podía la gente morir de eso en una época muy remota? ¿Cómo explicar la desaparición de la lepra en Europa, a finales de la Edad Media, cuando las sociedades europeas no contaban con ningún tratamiento? ${ }^{7}$

La idea radical según la cual los hallazgos de la ciencia solamente conciernen a realidades que comienzan a existir desde el momento de su descubrimiento, no es para nada aplicable a las realidades -difíciles pero no imposibles de conocer-que se pueden englobar bajo el nombre de historia natural de las enfermedades

7 Sobre este tipo de interrogaciones científicas véase, por ejemplo Donoghue (2005, p. 389394). 
infecciosas, un campo que interesa en especial a la epidemiología histórica, cuyo gran atractivo se basa en la pregunta arqueológica por las causas de enfermedad colectiva en sociedades ya desaparecidas, sobre todo para comprender la convivencia entre microorganismos patógenos, hábitats y sociedades humanas (GRMEK, 1993). Para el caso de la tuberculosis, ya hay avances que se refieren principalmente a la detección de huellas cadavéricas de la enfermedad en épocas muy antiguas o en la Edad Media. ${ }^{8}$

\section{La tuberculosis entre realidad biológica y representación}

Re-presentar - o hacer presente lo ausente mediante mecanismos simbólicos diversos como los usos de la imagen, del lenguaje y del discurso - constituye una facultad humana estrechamente ligada a la gestión de nuestra memoria colectiva y de nuestras formas de enriquecerla. Nada se puede conocer sin el acto de representar. En cierta forma, conocer es hacer inventarios gestionables de lo conocido, lo cognoscible e incluso, en ocasiones, de lo desconocido y de lo que no estamos en capacidad de conocer. Estos gestos y acciones se encaminan a mitigar la angustia que nos generan los misterios de la vida, del mundo y de nosotros mismos, pero también nos fuerzan a - o nos permiten — elaborar representaciones y, entre ellas, las que pueden agruparse bajo el sintagma representar la enfermedad..$^{9}$ Este puede servir para nombrar un conjunto muy complejo de prácticas discursivas y no

8 Hay que resaltar los excelentes estudios de antropología biológica sobre tuberculosis precolombina con los que ya se cuenta en Colombia: Hugo Armando Sotomayor Tribín, José Vicente Rodríguez Cuenca y Abel Fernando Martínez Martín, entre otros.

9 "Representar la enfermedad" remite a un problema muy complejo, pues no se trata solamente del esfuerzo médico y científico de definición y delimitación de la enfermedad, para distinguirla, nombrarla y clasificarla, es decir, para dominarla. El problema de la enfermedad desborda el campo de la medicina moderna, pues en tanto fenómeno humano exige representaciones, no solamente como esfuerzos de formulación más o menos coherentes, sino también como interpretación y búsqueda de sentido, lo que va más allá de la objetivación e involucra además la experiencia sensible y la subjetividad. Así, la representación de la enfermedad se basa en la necesidad que tenemos como humanos de vivir la enfermedad, más que como desorden, caos y azar, como acontecimiento cargado de significación, es decir susceptible de comprensión. A este respecto, ver los trabajos de Herzlich (1969) y Sontag (1996). 
discursivas que moldean los imaginarios individuales y colectivos ligados a la enfermedad.

El positivismo y el cientificismo del siglo XIX pretendieron separar de manera tajante entre formas "científicas" de representar los fenómenos de la naturaleza y las formas, por decirlo así, "salvajes" o "populares". Esta toma de posición ideológica también llegó a afectar los análisis de las formas de representación de las enfermedades, pero el trabajo crítico inaugurado por Ludwik Fleck a comienzos del siglo XX ha elevado la discusión a un nivel nada dogmático. Más recientemente, Claudine Herzlich (1969), Serge Moscovici (1993) y Susan Sontag (1996), entre muchos otros observadores, han mostrado que nuestra relación con la enfermedad, el dolor, el sufrimiento y la muerte produce imágenes -discursivas e icónicas- que difícilmente pueden separarse entre objetivas y subjetivas, sabias e ignorantes o en científicas y populares. Por su parte, Mirko D. Grmek introdujo desde 1969 el enfoque sintético de la historia de las enfermedades, que estudia las interconexiones sincrónicas entre diferentes enfermedades en una época precisa y los cambios diacrónicos de los conjuntos que él llama "patocenosis" (GRMEK, 1989).

$\mathrm{Ni}$ en la filosofía, ni en la sociología de la enfermedad, ni en la historia de la medicina, ni en la historia social y cultural de las enfermedades ha habido originalmente pretensiones de negación de la realidad biológica constituida por las enfermedades. Admitir que ellas no son solamente "representaciones" tampoco equivale a negar los aspectos simbólicos y sociales. Pero en este punto hay que situarse en el problema tal y como lo recibimos de las investigaciones y los debates de la segunda mitad del siglo XX y

10 El concepto de enfermedad es tomado aquí en una acepción integral que designa los cambios en el organismo humano que nos recuerdan nuestro cuerpo, nuestra capacidad de enfermar y de alcanzar un nuevo estado de normalidad, así como nuestro riesgo constante de morir. Del problema de las definiciones de salud y enfermedad se han ocupado, seriamente, por un lado, filósofos como Georges Canguilhem (1990) y Sandra Caponi (1997). Por otro lado, sociólogos y antropólogos: Herzlich (1969; 1984), George Foster y Barbara Gallatin Anderson (1978). Finalmente historiadores de la medicina y de la salud como George Rosen (1993), o los ya citados textos de Mirko Grmek. 
abandonar una supuesta jerarquía de los discursos, las imágenes y las representaciones sobre la enfermedad. Para las ciencias sociales, en general, y para la historia, en particular, están en el mismo nivel de importancia las diversas representaciones de la salud y la enfermedad (las del paciente, las mediáticas, las académicas), en la medida en que todas pueden ser estudiadas desde los puntos de vista socio-antropológico e histórico.

\section{La representación "tuberculosis enfermedad social" en Colombia}

No cabe duda de que en Colombia el quiebre reciente en la historiografía de las ciencias, la medicina, la salud y la enfermedad proviene del impulso que, desde la década de 1980, investigadores como Emilio Quevedo, Olga Restrepo y Diana Obregón le han dado a la incorporación de preguntas de las ciencias sociales y de la epistemología, por la cual se ha llegado a superar una forma tradicional de interrogación anecdótica, cronista y acontecimental. Este cambio y las nuevas tendencias están bien descritos en el balance historiográfico que hace la historiadora y médica Magnolia Arango en su tesis de una historia de la tuberculosis en Bogotá a través de la práctica médica en el Hospital San Juan de Dios, durante la transición del siglo XIX al siglo XX (ARANGO LOBOGUERRERO, 2007). Aunque la autora se centra en las concepciones desplegadas por los médicos, su investigación es uno de los pocos estudios que se interesan por la historia de las representaciones de la tuberculosis en Colombia. Según sus hallazgos, se puede afirmar que la representación médica y universitaria de la tuberculosis en Colombia estuvo en sintonía con el conjunto de debates y discusiones que se dieron en el mundo europeo durante el siglo XIX, pero sobre todo durante el periodo 1870-1950. Estas fechas aproximadas abarcan desde los comienzos de una enseñanza médica formalizada en Colombia, que otorgaba títulos y regulaba cierta parte de la producción y reproducción de conocimientos médicos mediante el requisito de la tesis, hasta los comienzos de la generalización de los tratamientos quirúrgicos y antibióticos en centros especializados 
en enfermedades del pulmón.

La investigadora Magnolia Arango muestra que el debate sobre la etiología de la tuberculosis también estuvo ligado a tres "paradigmas" médicos: el de la etiología mecanicista o de terreno, de corte neohipocrático, según el cual las causas de la enfermedad debían buscarse sobre todo en las condiciones de vida de los pobladores y en las cualidades salubres o insalubres de sus lugares geográficos; el del debate de la herencia, con todas sus ambiguiedades, según el cual la predisposición a ser tuberculizable se podía heredar, pero también se podía adquirir a causa de condiciones miserables e insalubres y como consecuencia de vicios y hábitos libertinos como el alcoholismo; el tercer bloque paradigmático lo constituía la discusión acerca del contagio, concepción minoritaria, hasta cuando, en 1865, J. A. Villemin logró enfermar de tuberculosis animales de laboratorio y, en 1882, Robert Koch aisló el bacilo causal.

La investigadora muestra que estos avances médicos no desplazaron una "mentalidad anatomoclínica" para instalar otra nueva, la microbicista (ARANGO LOBOGUERRERO, 2007, p. 251). En efecto, respecto a la etiología, después de Pasteur, Villemin y Koch, en los estudios médicos colombianos sobre tuberculosis no se eligió una línea teórica precisa. En realidad, según la misma autora, la atención a las condiciones de vida y al terreno nunca fue abandonada, aunque ya se conociera un agente causal biológico. Más bien, la hipótesis del contagio, reforzada por la teoría de los gérmenes, se unió a la de las condiciones de terreno y juntas formaron la vía para estigmatizar la pobreza como fuente de enfermedad y la enfermedad como fuente de degeneración y de prolongación de la miseria. A esta etiología se sumó rápidamente una especie de causalidad moral que comenzó a señalar la "intemperancia" y los "vicios" como generadores de terrenos propicios para el contagio. Sobre estas bases etiológicas políticos, reformadores, higienistas y médicos forjaron rápidamente - tanto en Europa como en Colombia-, durante la segunda mitad del siglo XIX y las primeras décadas del siglo XX, la representación de la tuberculosis como 
enfermedad social o como la enfermedad social por excelencia. La configuración de esa representación tuvo gran asiento en los procesos acelerados de urbanización e industrialización vividos en Europa durante el siglo XIX, y en otros lugares del mundo, con ciertos desfases de tiempo, durante el siglo XIX y la primera mitad del siglo XX.

Las concepciones y los debates científicos y políticos europeos sobre la tuberculosis habían alcanzado un alto grado de madurez y de síntesis ${ }^{11}$ en el momento en que, en Colombia, entre 1913 y 1916, en el ámbito político, se tomaban decisiones sobre la "lucha antituberculosa" (REPÚBLICA DE COLOMBIA, 1917). Por su parte, varios médicos colombianos ya habían comenzado a alertar sobre esta amenaza sanitaria como "problema social" o como "enfermedad social", sobre todo desde la última década del siglo XIX (DUQUE, 1899; ISAZA, 1915). De ahí que el comienzo de la lucha antituberculosa como campaña oficial, en 1916, estuviera inspirado en el pleno auge y características de la representación de la tuberculosis como "enfermedad social".

Para tratar los aspectos sociales y culturales de la discusión sobre la etiología de la tuberculosis el discurso médico es muy esclarecedor, pero no es suficiente. De entrada, se puede enunciar como hipótesis de trabajo que, en Colombia, como en otros lugares del mundo por la misma época, la generalización de la concepción médica de la tuberculosis como enfermedad contagiosa, infecciosa, evitable y social hizo de ella un medio de legitimación del discurso y las prácticas de higiene privada y pública y, por esa vía, del poder creciente de los médicos mas allá de la universidad.

La discusión que comenzó en Colombia en las dos últimas décadas del siglo XIX no se centró en el carácter contagioso de la enfermedad. Eso se debió en parte a una especie de consenso pasteuriano, como si de verdad dominara un paradigma microbicista. Pero esto no es tan exacto. No hubo "paradigma" ni "mentalidad"

11 Prueba de ello son los numerosos y casi rutinarios congresos internacionales de la tuberculosis: París 1898; Berlín 1899; Londres 1901; París 1905; Washington 1908; Berlín 1912. Véase: historiadelamedicina.org, 2010. 
dominante, o no dominaba enteramente uno u otra. Más bien hubo una mixtura ecléctica, pero no caótica, de diversas teorías. Al menos tres: la climática y del terreno (mecanicista), la del contagio (vitalista, o al menos biologicista) y la de la herencia (más que todo social). En cuanto a la primera, consistía en señalar los terrenos, las secciones espaciales y los climas más susceptibles de propiciar la infección tuberculosa; la segunda se basaba en la verificación de la existencia del bacilo y que se cumplieran los postulados de Koch; la tercera no era para-genética, sino que atendía a que la predisposición a ser tuberculizable podía ser heredada. En realidad, las tres concepciones se mezclaban y complementaban, no se oponían. En los campos político y social, unir estas tres concepciones equivalía a señalar una enfermedad contagiosa sin mucho daño a las ideas liberales, para las cuales el comercio era como el sistema circulatorio de la nación, es decir "vital". Para las élites colombianas de finales del siglo XIX y comienzos del XX señalar amenazas de contagio era siempre un riesgo terrible de aislamiento respecto del comercio marítimo internacional. En el interior del país, la concepción triple permitió emprender ciertas geografías del mal, emulando la que hiciera, en 1885, para la ciudad de París, el médico higienista francés Jacques Bertillon (1885), y además señalar, dadas las precarias condiciones de vida de estos grupos sociales, a los obreros, los pobres, los presos, los alienados, los indigentes y los militares como focos de infección. Por último, haber añadido la idea de herencia de la susceptibilidad tuberculizable permitió señalar a los alcohólicos y a todos los "intemperantes" como focos de infección, pues eran personas débiles susceptibles de infectarse y de transmitir a su progenie la tal predisposición.

No es un azar que Colombia tampoco escapara a la compleja y expandida representación de la tuberculosis como enfermedad social. La mezcla lógica de concepciones sobre la etiología de la enfermedad que caracterizó a esta representación durante el periodo 1882-1943 (microbicista y sanatorial) no provocó una "cruzada" filantrópica para mejorar las condiciones de vida de los pobres, sino que reforzó la política segregacionista basada en la idea de localizar la enfermedad entre ellos, aislar a los enfermos y contrarrestar de este modo su propagación. 
Con esta representación de la enfermedad, la tuberculosis y su profilaxis se convirtieron en argumentos fuertes para que la higiene pública como discurso moral, médico y educador pudiera legítimamente penetrar con su poder en la vida privada. Así, la intervención higienista en los espacios públicos y privados era una manera de paliar las contradicciones entre el crecimiento del poder coercitivo del Estado y las ideas liberales de respeto a la libertad individual y de empresa, y por esa vía mantener el carácter vago de las fronteras entre lo privado y lo público.

Es claro que en la historia de la tuberculosis en Colombia nos enfrentamos a una fuerte representación médico-social que puede haber tenido usos y abusos políticos todavía por explorar. Sin embargo, esto no nos puede hacer olvidar que los aspectos humanos y biológicos, incluso propiamente médicos, como son las realidades del dolor, el sufrimiento y la muerte ligados a esta enfermedad, no se pueden enfrentar solamente como si fueran otras formas de representarse la enfermedad. Esta sensibilidad de la investigación histórica frente a los aspectos corporales y biomédicos de la enfermedad plantea como necesaria una historia de la tuberculosis desde el punto de vista de las historias de vida de los pacientes, lo que exige salir de los archivos oficiales y médicos y consultar los judiciales, los privados, los epistolares, los hospitalarios y los fondos de solicitudes y quejas.

Tampoco debemos olvidar que en la complejidad de la representación social "tuberculosis" también interviene la prolongada impotencia terapéutica ante la enfermedad. Hubo un punto de quiebre en plena Segunda Guerra mundial y desde comienzos de la posguerra, cuando comenzó a expandirse en el mundo el uso de los antibióticos. Parece que no muy rápido en Colombia, pero comienza de todas maneras una nueva era para el tratamiento de la tuberculosis. Lo que haya pasado en este campo aun se desconoce. Como tampoco se ha explorado esta historia a partir de la pregunta por la efectividad de las terapéuticas de la época anterior a la aparición de los antibióticos (incluidas las quirúrgicas como el neumotórax). 


\section{Emergencia de los argumentos cuantitativos}

El periodo histórico durante el cual emerge, se configura y se estabiliza en Colombia la representación de la tuberculosis como enfermedad social (aproximadamente entre 1915 y 1951), comprende también una serie de acontecimientos ligados a la emergencia de lo que oficialmente en la época se llama "estadística médica”. A partir de la Ley 63 de 1914, sobre reorganización del Servicio Nacional de Estadísticas, se propuso una reforma de la estadística nacional que incluyó como campos obligatorios la "mortalidad", la "morbilidad" y la "estadística nosográfica", que debían establecerse según el sistema de Jacques Bertillon (1896). Se instauró entonces un mecanismo permanente de gobierno, que comprometía a funcionarios públicos (alcaldes y médicos) en la recolección de los datos que conformaran la "estadística médica", alimentada por datos exigidos a funcionarios públicos (alcaldes y médicos).

Al mismo tiempo, tomó fuerza la exigencia de la función médica oficial para cada municipalidad. ${ }^{12}$ Aunque no hubiera personal diplomado ni dinero para hacer cumplir este requerimiento, se intentó de manera insistente, a partir de 1915, instaurar esta función en los pueblos, ya que en las ciudades capitales ya estaba establecida desde 1914.

Se trata de acontecimientos significativos del proceso de medicalización de la vida y la sociedad en Colombia, pues muchos problemas y asuntos de la vida cotidiana, ya subrayados por los higienistas de finales del siglo XIX, se convirtieron en objetivos

12 Mediante la Ley 53 de 1914 se estableció el servicio nacional de medicina legal. La necesidad de normalizar esta práctica fue una poderosa razón para exigir la presencia de médicos oficiales, pero no la única. La Ley 86 de 1914, que deroga la Ley 33 de 1913 y que organiza la higiene y la salubridad en la nación, refuerza la importancia de los médicos higienistas. La Ley 32 de 1918 planteó una nueva reforma, creó la Dirección Nacional de Higiene, estableció que "las disposiciones tomadas por el director no estaban sujetas a la aprobación de ninguna otra autoridad y tenían fuerza de ley” (QUEVEDO, 2003, p. 102). La Ley 32 de 1918 insiste sobre la necesidad de médicos en zonas rurales y pueblos para el ejercicio de la medicina legal, la asistencia médica a los pobres y la administración de la higiene y la salubridad. 
del saber y del poder médico en la segunda década del siglo XX. La adopción de la nomenclatura y los métodos estadísticos de Jacques Bertillon en ese momento fue un punto de llegada tras debates y discusiones, pero también uno de los episodios que muestran la importancia que los higienistas de finales del siglo XIX y comienzos del siglo XX le dieron a la estadística médica. De nuevo, aquí sólo hay preguntas que apenas comenzarán a resolverse con investigaciones: ¿Cómo interpretar la queja ritual de los médicos higienistas, desde finales del siglo XIX y hasta muy entrado el siglo $X X$, acerca de la inmensa necesidad de organizar en Colombia estadísticas de morbilidad y mortalidad y las grandes dificultades para levantar los datos? ¿Cuál fue el papel de los argumentos cuantitativos en la conformación de la representación de la tuberculosis como enfermedad social? Una lectura cruzada de las cifras y los discursos sobre la tuberculosis de finales del siglo XIX y comienzos del siglo XX, podría ayudar a responder si se trató de un verdadero problema sanitario y colectivo, en términos biomédicos y epidemiológicos, o si formó solamente un conjunto de representaciones sociales y culturales basadas en el estigma sociocultural atribuido a esta enfermedad en ese periodo.

\section{Preguntas finales}

La poca bibliografía colombiana, la abundante bibliografía internacional y una primera mirada a las fuentes locales sugieren otros problemas, que aquí solamente se enumeran en forma de preguntas: ¿Produjeron resultados -y cuáles- las acciones de prevención anteriores a la aparición de los antibióticos? ¿Qué pasó con los problemas de ocultación del diagnóstico, persecución a enfermos y luchas de resistencia de los enfermos? Ante la prolongada impotencia terapéutica, ¿qué habrá pasado con el "florecimiento del charlatanismo" estudiado por Pierre Guillaume para el caso francés? ¿Qué más podremos saber de la exclusión de las personas señaladas como "tuberculosas", de las curas sanatoriales, del trabajo de los dispensarios, de las visitas médicas domiciliares para localizar y someter a los enfermos? ¿Qué sabremos sobre el 
funcionamiento de la declaración obligatoria de la enfermedad? ¿Qué pasó en el campo de la producción y el comercio de vacunas y sueros preventivos? ¿Hubo en Colombia, como en otros países, debates sobre todos estos aspectos?

Recebido: 29.06.2011

Aprovado: 31.08.2011

\section{Fuentes históricas}

BERTILLON, J. De l'augmentation de fréquence des principales maladies épidémiques à Paris, pendant la période 1865-1883, et de leurs saisons d'élection. Paris: Imp. Municipale, 1885.

- Cours élémentaire de statistique administrative: Élaboration des statistiques-organisation des bureaux de statistique-éléments de démographie. Paris: Société d'Éditions Scientifiques, 1896.

CONCEJO DE MEDELLín. Acuerdo $n^{\circ} 4$ de enero de 1920 sobre Junta Municipal Organizadora de la Lucha Antituberculosa. Crónica Municipal Medellín v. IX, n. 265 a 268, p. 21, ene. 1920.

- Felicitación al Concejo [Hospital La María]. Crónica Municipal Medellín v. XII, n. 383, p. 3023, oct. 1923.

DUQUE, J. M. Contribución al estudio de la tuberculosis en Antioquia. Tesis Facultad de Medicina y Cirugía Universidad de Antioquia. Medellín: Imprenta del Departamento, 1899.

ISAZA S. E. La tuberculosis en Bogotá, profilaxis. Tesis Facultad de Ciencias Naturales y Medicina, Universidad Nacional Colombia. Bogotá: Arboleda y Valencia, 1915.

REPÚBLICA DE COLOMBIA. Disposiciones sobre la lucha antituberculosa en Colombia. Bogotá: Imprenta Nacional, 1917.

Bibliografía general

ARMUS, D. La ciudad impura: salud, tuberculosis y cultura en Buenos Aires, 1870-1950. Buenos Aires: Edhasa, 2007 
BRYDER, L. Below the Magic Mountain. A Social History of Tuberculosis, in Twentieth-Century Britain. Oxford: Clarendon E'ress, 1998.

CANGUILHEM, G. "La salud, concepto vulgar y problema filosófico". Sociología v. 21, p. 151-158, jul. 1998. Trad. Jorge Márquez Valderrama.

CAPONI, S. Georges Canguilhem y el estatuto epistemológico del concepto de salud. História, Ciências, Saúde - Manguinhos v. IV, p. 287-307, jul.-oct. 1997.

CARBONETTI, A. Enfermedad y sociedad: la tuberculosis en la ciudad de Córdoba 1906 y 1947. Argentina: Municipalidad de Córdoba, 1998.

CENTRE MÉDICAL DE BLIGNY. Bligny et son histoire: du sanatorium au centre médical d'aujourd'hui. Document numérique du Centre Médical de Bligny (2008). Conception, réalisation: Imprimerie BP. http://www.cm-bligny.com/IMG/pdf/Bligny_historique_2008_web_ size.pdf

DESSERTINE, D; FAURE, O. Combattre la tuberculose. 1900-1940. Lyon: Presses Universitaires de Lyon, 1988.

DONOGHUE, H.D. (org.). Co-Infection of Mycobacterium tuberculosis and Mycobacterium leprae in Human Archaeological Samples: A Possible Explanation for the Historical Decline of Leprosy. Proceedings: Biological Sciences of The Royal Society v. 272, n. 1561, p. 389-394, feb. 2005.

FLECK, L. La génesis y el desarrollo de un hecho científico: introducción a la teoría del estilo de pensamiento y del colectivo de pensamiento. Madrid: Alianza, 1986.

.Problèmes de théorie des sciences. Archives de philosophie v. 73, n. 4, p. 585-600, 2010.

FOSTER, G.M.; ANDERSON, B.T. Medical Anthropology. New York: John Wiley and Sons, 1978.

GRMEK, M.D. Préliminaires d'une étude historique des maladies. Annales. Histoire, Sciences Sociales v. 24, n. 6, Histoire biologique et Société, p. 1473-1483, nov. 1969. 
. La malattia come concetto e come effettualità. Inserviste. http://www.emsf.rai.it/interviste/interviste.asp?d=442\#1, 26 de jun., 1989.

Le concept de maladie émergente. History \& Philosophy of the Life Sciences v. 15, n. 3, p. 281-296, 1993.

. ; SOURNIA, J. Les maladies dominantes. In Mirko, M.D. (org.). Histoire de la pensée médicale en Occident Du romantisme à la science moderne vol 3. Paris: Seuil, 1999.

GUILLAUME, P. Du désespoir au salut: les tuberculeux aux XIXe et XXe siècles. Paris: Aubier, 1986.

Histoire d'un mal, histoire globale. Du mythique à l'économique. In Bardet, $\mathrm{J}$ (org.). Peurs et terreurs face à la contagion. Paris: Fayard, 1988.

HERZLICH, C. Santé et maladie: analyse d'une représentation sociale. Paris, La Haye, Mouton et Cie, 1969.

. La problématique de la représentation sociale et son utilité dans le champ de la maladie. Sciences sociales et santé v. 2 , n. 2, p. 71-84, 1984.

LATOUR, B. Jusqu'où faut-il mener l'histoire des découvertes scientifiques? Ramsès II est-il mort de la tuberculose?. La Recherche 309, p. 72-73, mar. 1998.

MOLERO MESA, Jorge. Estudios médicosociales sobre la tuberculosis en la España de la restauración. Madrid: Ministerio de Sanidad y Consumo, 1987.

MOSCOVICI, S. La perception quotidienne de la santé et de la maladie: théories subjectives et représentatives sociales. Paris: L'Harmattan, 1993.

NASCIMENTO, D. Preventório Rainha Dona Amélia: um sanatório para crianças enfraquecidas. Asclepio. Revista de Historia de la Medicina y de la Ciencia v. 60, n. 2, p. 143-166, jul.-dic. 2008.

OBREGÓN, D. Batallas contra la lepra: Estado, medicina y ciencia en Colombia. Medellín: Universidad Eafit, 2002.

PORTO, À. Tuberculose: a peregrinação em busca da cura e de uma 
nova sensiblidade. In: Nascimento, D. y Carvalho, D.M. (org.). Uma história brasileira das doenças. Brasilia: Paralelo 15, 2004.

QUEVEDO, E. Historia social de la ciencia en Colombia. La institucionalización de la medicina en Colombia. Bogotá: Colciencias, 1993.

. (org.). Café y gusanos, mosquitos y petróleo: El tránsito de la higiene hacia la medicina tropical y la salud pública en Colombia 1873-1953. Bogotá: Universidad Nacional, 2004.

RODRÍGUEZ OCAÑA, E. Por la salud de las naciones: higiene, microbiología y medicina social. Madrid: Akal, 1992.

ROSEN, G. A history of public health. Baltimore, London: John Hopkins University Press, 1993.

SILVEIRA, A.T; NASCIMENTO, D. A doença revelando a história. Uma historiografia das doenças". In: Nascimento, D. y Carvalho, D.M. (org.). Uma história brasileira das doenças. Brasilia: Paralelo $15,2004$.

SONTAG, S. La enfermedad y sus metáforas/El sida y sus metáforas. Madrid: Taurus, 1996.

TELLER, M.E. The Tuberculosis Movement. A Public Health Campaign in the Progressive Era. New Cork: Greenwood Press, 1988.

Anexo: Historiografía de la tuberculosis en Colombia

ARANGO, A. R. Introducción a la historia de la lucha contra la tuberculosis en Medellín a principios del siglo XX. Trabajo de grado en Historia, Universidad Nacional de Colombia, sede Medellín, 2010.

ARANGO LOBOGUERRERO, M. Apuntes sobre la historia de la tuberculosis infantil. Medicina, Academia Colombiana de Medicina v. 25, n. 2, p. 122-127, 2003.

La tuberculosis en la transición del siglo XIX al XX: la evolución de los conceptos sobre la enfermedad: el caso de Bogotá visto a través del Hospital San Juan de Dios. Tesis de maestría, Universidad Nacional de Colombia-Bogotá, 2007. 
CARDONA, Á. Problemática médica antioqueña y su marco sociopolítico en la primera mitad del siglo XX. Medellín: Universidad de Antioquia, 1984.

CONGOTE, J.C. Las enfermedades sociales en los obreros de Medellín 1900-1930. Monografía de Historia, Medellín, Universidad de Antioquia, 2007.

HERNÁNDEZ, M.; MALDONADO, H. Memorias de un sanatorio antituberculoso. Biomédica v. 24, n. 1, p. 27-33, 2004.

IDROVO, Á.J. Tuberculosis prehispánica en muiscas de la Sabana de Bogotá. Rev Fac Med Univ Nacional, v. 45, n. 1, p. 50-54, 1997.

Notas sobre el inicio de la epidemia de tuberculosis pulmonar en Bogotá (1870-1920). Biomédica, v. 21, n. 3, p. 216223, 2001.

Raíces históricas, sociales y epidemiológicas de la tuberculosis en Bogotá, Colombia. Biomédica, v. 24, n. 4, p. 356$365,2004$.

MARTÍNEZ, A.F.; MELÉNDEZ, B.F;; GAMBOA, L.P. Un posible caso de tuberculosis en una momia muisca. Colombia Iatros Revista Médica Estudiantil v. 4, n. 1, p. 50-59, 2006.

OCAMPO, M.B. Contagio y enfermedad social tratamiento sociopolítico e imaginario en torno a una patología colectiva: tuberculosis en Medellín 1923-1959. Trabajo de grado en Historia, Universidad Nacional de Colombia, Medellín, 2005.

OSPINA, S. La tuberculosis, una perspectiva históricoepidemiológica. Infectio, Revista de la Asociación Colombiana de Infectología, v. 5, n. 4, p. 241-250, 2001.

RODRÍGUEZ CUENCA, J.V. Las enfermedades en las condiciones de vida prehispánica de Colombia. Bogotá: Universidad Nacional de Colombia, 2006.

SOTOMAYOR, H; BURGOS, J.; ARANGO, M. Demostración de tuberculosis en una momia prehispánica colombiana por la ribotipificación del ADN de Mycobacterium tuberculosis. Biomédica, v. 24, n. 1, p. 18-26, jun. 2004. 
VÉLEZ, M.S. Medellín en los años veinte: la industrialización y la fundación del Hospital La María. Monografía de Historia. Medellín: Universidad de Antioquia, 2001.

\begin{abstract}
Towards a history of the tuberculosis control in Colombia

The problem of social and cultural history of tuberculosis control in Colombia has hardly been contemplated by researchers. A cross-reading of figures and discourses on tuberculosis in the late nineteenth and early twentieth centuries would help answer the question: Was it actually a collective health problem, in biomedical and epidemiological terms, or was it merely a set of social and cultural representations based on the stigma attached to this disease? On the historiographic approach, this article assesses what has been done in this field in order to propose new research strategies, treating tuberculosis control as a socio-cultural problem in the history of health policies and health reforms in Colombia between 1913 and 1950. On the filing approach, an emphasis on published literature and files on campaigns, prevention, treatment, hygiene, health care and the eugenics discussion is made.
\end{abstract}

Keywords: history of tuberculosis-Colombia, medicalization processes, social representation of illness, history of medicine, history of medicineColombia. 